\title{
CLINICAL OUTCOMES AFTER RIDGE PRESERVATION WITH TWO XENOGRAFTS: A 4-YEAR RANDOMIZED CLINICAL TRIAL
}

IMPLANT THERAPY OUTCOMES, SURGICAL ASPECTS
Galletti, Guido4; Borgia, Valentina4; Alfonsi, Fortunato 3,2 ; Covani, Ugo'1,2; Barone, Antonio4.

1) University of Pisa, Italy; 2) Tuscan Stomatologic Institute, Italy; 3) University of Florence, Italy; 4) University of Geneve, Switzerland

Abstract
After tooth extraction the alveolar ridge undergoes horizontal and vertical resorption that may
lead to an unaesthetic outcome. This is why several techniques have been proposed for ridge
preservation after tooth extraction. One of the most accepted is the placement of a bone graft.
Aim of this study was to evaluate and compare at 4 years follow-up clinical and esthetic
outcome of single-implant restorations placed after socket preservation grafted with cortical
porcine bone vs collagenated cortico-cancellous porcine bone and spontaneously healed
sockets. Thirty-four patients in the present randomized controlled clinical trial underwent
single-tooth extraction in the premolar/molar areas. 10 sites were grafted with collagenated
cortico-cancellous porcine bone(coll), 10 sites with cortical (cort) porcine bone and 14 sites
underwent natural healing(control). Four months after tooth extraction and ridge preservation
implants were placed. Prosthetic restorations were delivered after a 4-month period of healing.
Clinical and aesthetical parameters were evaluated for a follow-up period of 4 years. Both test
groups showed better outcome in terms of facial soft tissue level, papilla index and marginal
bone resorption than the control group. Favorable esthetic outcome and stability of soft/hard
tissue around single implant crowns can be expected in patients with ridges preserved either
with cortical or collagenated cortico-cancellous porcine bone.

\section{Background and Aim}

After tooth extraction the alveolar ridge undergoes both horizontal and vertical resorption. Several studies reported that horizontal bone resorption is about $63 \%$ while the vertical bone resorption is about $22 \%^{1}$. Taking in analysis soft tissue volume highly increases the rate of both horizontal and vertical resorption 2,3 . These physiological events may influence the future outcome of the implant treatment, leading to an unsatisfactory aesthetic result. This is why several techniques have been proposed for ridge preservation after tooth extraction ${ }^{4}$. Studies attested that tissue changes after tooth extraction could be reduced by grafting the socket with biomaterials ${ }^{5}$. Extraction sockets grafted with xenograft materials have shown significant reduction of ridge changes compared with sockets that had spontaneous healing ${ }^{6,7}$. However, the amount of ridge modifications after socket grafting have shown different results, which can be related to the type of biomaterial used and the residual buccal bone thickness 8 . Many studies demonstrated that cortical or cancellous bone substitutes have different healing patterns. For these reasons a proper knowledge over the biomaterials used for socket preservation seems to be fundamental, in order to guarantee to the patient the best aesthetic outcome of the implant therapy.

\section{Methods and Materials}

$\checkmark 34$ patients were enrolled in the present randomized controlled clinical trial and underwent single-tooth extraction in the premolar/molar areas.

$\checkmark 10$ sites (coll) were grafted with collagenated cortico-cancellous porcine bone (MP3, OsteoBiol ${ }^{\circledR}$, Tecnoss ${ }^{\circledR}$, Coazze, Italy)

$\checkmark 10$ sites (cort) with cortical porcine bone (APATOS, OsteoBio| ${ }^{\circledR}$, Tecnoss ${ }^{\circledR}$, Coazze, Italy) $\checkmark 14$ sites underwent natural healing (control)

$\checkmark 4$ months after tooth extraction and ridge preservation implants were placed. Prosthetic restorations were delivered after a 4-month period of healing.

$\checkmark$ Clinical and radiological measurements were registered at 1 Year and 4 Years after prosthetic delivery.

The outcome variables used for the follow-up were:

Peri-implant marginal bone level (MBL) was set as the distance between the reference point and the most apical point of the MBL. The reference point was the fixture-abutment interface. evaluated on intraoral radiographs at the mesial $(m)$ and distal $(d)$ sites (mMBLX and $d M B L X$, in which $X=0,1,2$, and 3 for times $T_{0}, T_{1}, T_{2}$, and $T_{3}$, respectively)

Facial soft tissue level (FSTL) was evaluated by measuring the distance between the level of soft tissue at the mid-facial gingival level and a reference line, which connected the FSTL of the adjacent teeth

Papilla Index (PI) was evaluated in accordance to Jemt classification" giving a value to the two papillary scores (mesial and distal): absence of papilla (score 0 ), less than half of the height of the papilla (score 1), at least half of the height of the papilla (score 2), the papilla fills up the entire space (score 3), the papilla is hyperplastic (score 4). An average between the 2 scores (mesial and distal) was done for each tooth

All analyses were collected and measured by an independent and well experienced observer. Together with descriptive statistics, for each of the outcome variables, pairwise comparisons were performed using the Wilcoxon signed-rank test for matched samples. The level of statistical significance was set at 0.01 for all analyses.

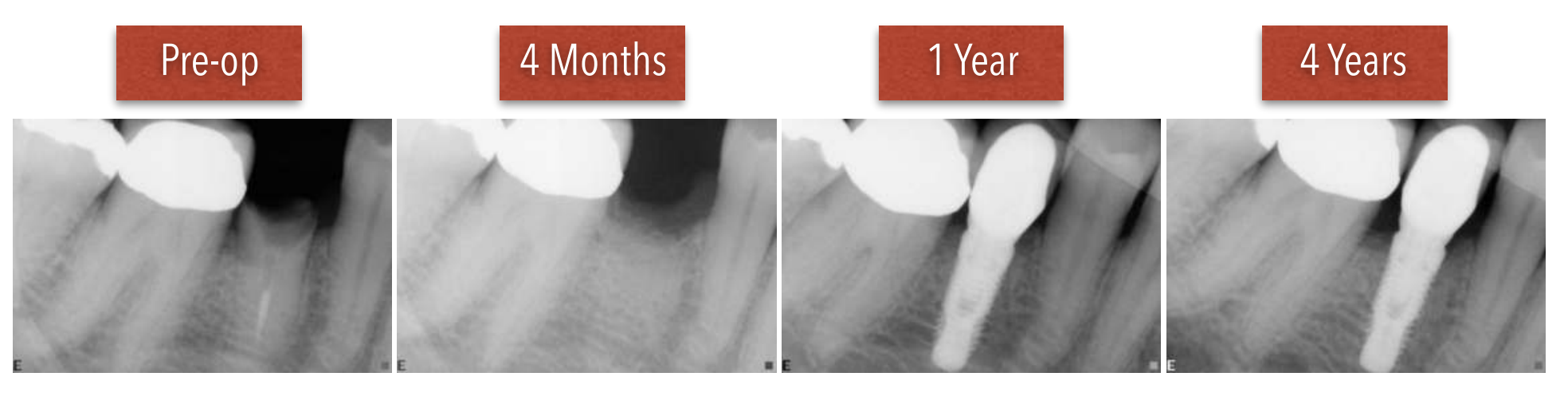

Results

\begin{tabular}{|c|c|c|c|c|c|c|c|c|c|c|c|}
\hline \multirow{3}{*}{$\begin{array}{c}\text { variables } \\
\text { time }\end{array}$} & \multicolumn{5}{|c|}{ groups } & \multicolumn{6}{|c|}{ statistics (P-value) } \\
\hline & collagenated (col) & \multicolumn{2}{|c|}{ cortical (cor) } & \multicolumn{2}{|c|}{ control (con) } & \multicolumn{3}{|c|}{$1 \mathrm{yr}$} & \multicolumn{3}{|c|}{ 4yrs } \\
\hline & 4yrs & $1 \mathrm{yr}$ & 4yrs & $1 \mathrm{yr}$ & $4 y r s$ & col vs cor & col vs con & cor vs con & col vs cor & col vs con & cor vs con \\
\hline$\Delta \mathrm{MBL}(\mathrm{mm})$ & $-0.54 \pm 0.46$ & $-0.29 \pm 0.4$ & 1+1+ & $-0.78 \pm 0.52$ & $.71 \pm 0.5$ & 0.16311 & 0.19775 & 0.01343 & 0.18525 & 0.00086 & 0.00001 \\
\hline$\Delta$ FSTL (mm) & $0.36 \pm 0.63$ & $-0.08 \pm 0.5$ & $0<0$ & $-0.92 \pm 0.67$ & $.38 \pm 0.99)$ & 0.06634 & 0.00002 & 0.00170 & 0.15586 & 0.00001 & 0.00032 \\
\hline PI & $1.25(1.25) \bigcirc 2.75(0.88$ & $1.5(0.5)$ & $(0.13$ & $2.0(0.5)$ & $.25(1.66)$ & 0.84169 & 0.00427 & 0.00060 & 0.07246 & 0.00395 & 0.00074 \\
\hline
\end{tabular}

\section{Conclusions}

Several studies has demonstrated a notable volume reduction after tooth extraction ${ }^{1}$. These changes could compromise the aesthetic outcome of final restoration, either with an implant-supported restoration 0 a traditional tooth-supported restoration. Although different techniques have been proposed for maintaining the original alveolar ridge dimensions, a complete preservation seems to be a non-achievable outcome ${ }^{10}$. Previous studies from Barone et al. demonstrated that the marginal bone level around implants placed in grafted sockets and in naturally healed sockets have similar clinical performances ${ }^{11}$, in this study the marginal bone level around implants placed in sockets grafted either with Coll or Cort seemed to be more stable than the no-grafted group. The long term stability of soft tissues (in terms of FSTL and $\mathrm{PI}$ ) is crucial for a positive aesthetic outcome. Outcomes from this study showed that facial soft tissue level remained stable at both 1 year and 4 years evaluation in the coll and cort group, conversely control group had some buccal recession. Maintenance of papillae height and appearance is crucial for a successful outcome of a prosthetic restoration. In both grafted group a satisfying regrowth of the papillae was achieved at 4 years evaluation, with a statistically significant difference compared to control group. This result might be related to the better sustenance of soft tissues by peri-implant hard tissue, that showed lower degree of bone resorption.

Within the limitations of this study, it can be concluded that more favorable esthetic outcome and stability of soft/hard tissue around single implant crowns can be expected in patients with ridges preserved either with cortical or collagenated cortico-cancellous porcine bone compared to spontaneous healing sockets, there was no significant difference between the 2 grafted groups

\section{References}

